\title{
On the characterization of equilibria of nonsmooth minimal-time mean field games with state constraints
}

\author{
Saeed Sadeghi Arjmand ${ }^{1}$ and Guilherme Mazanti ${ }^{2}$
}

\begin{abstract}
In this paper, we consider a first-order deterministic mean field game model inspired by crowd motion in which agents moving in a given domain aim to reach a given target set in minimal time. To model interaction between agents, we assume that the maximal speed of an agent is bounded as a function of their position and the distribution of other agents. Moreover, we assume that the state of each agent is subject to the constraint of remaining inside the domain of movement at all times, a natural constraint to model walls, columns, fences, hedges, or other kinds of physical barriers at the boundary of the domain. After recalling results on the existence of Lagrangian equilibria for these mean field games and the main difficulties in their analysis due to the presence of state constraints, we show how recent techniques allow us to characterize optimal controls and deduce that equilibria of the game satisfy a system of partial differential equations, known as the mean field game system.
\end{abstract}

\section{INTRODUCTION}

The concept of mean field games (referred to as "MFGs" in this paper for short) was first introduced around 2006 by two independent groups, P. E. Caines, M. Huang, and R. P. Malhamé [1], [2], and J.-M. Larsy and P.-L. Lions [3], [4], motivated by problems in economics and engineering and building upon previous works on games with infinitely many agents such as [5], [6]. Roughly speaking, MFGs are game models with a continuum of indistinguishable, rational agents influenced only by the average behavior of other agents, and the typical goal of their analysis is to characterize their equilibria. We refer the interested reader to [7] for more details on MFGs.

In this paper, we study an MFG model inspired by crowd motion in which agents want to reach a given target set in minimal time, their maximal speed being bounded in terms of the distribution of other agents and their state being constrained to remain in a given bounded set. Modeling and analysis of crowd motion have been the subject of a large number of works from different perspectives, such as [8][10], and some deterministic and stochastic MFG models have been already proposed, for instance, in [11]-[16]. MFG models for crowd motion usually try to capture strategic choices of the crowd based on the rational anticipation by an agent of the behavior of others.

\footnotetext{
${ }^{1}$ CMLS, École Polytechnique, CNRS, Université Paris-Saclay, 91128, Palaiseau, France, \& Université Paris-Saclay, CNRS, CentraleSupélec, Inria, Laboratoire des signaux et systèmes, 91190, Gif-sur-Yvette, France. saeed.sadeghi-arjmandepolytechnique.edu

${ }^{2}$ Université Paris-Saclay, CNRS, CentraleSupélec, Inria, Laboratoire des signaux et systèmes, 91190, Gif-sur-Yvette, France. guilherme. mazanti@inria.fr
}

The MFG model we consider in this paper is that of [15], its detailed description is provided in Section [I An important feature of the model from [15] which renders its analysis more delicate is the fact that the final time of the movement of an agent is not prescribed, but it is part of the agent's optimization criterion. Reference [15] establishes existence of equilibria of the considered MFG model, but additional properties, such as characterization of optimal controls and characterization of equilibria through the system of PDEs known as MFG system, are only obtained in [15] under the restrictive assumption that the target set of the agents is the whole boundary of the domain, which avoids the presence of state constraints in the minimal-time optimal control problem solved by each agent. The main contribution of the present paper is to characterize optimal controls and obtain the MFG system without such a restrictive assumption.

The major difficulty in analyzing optimal control problems with state constraints is that their value functions may fail to be semiconcave (see, e.g., [17, Example 4.4]), the latter property being important in the characterization of optimal controls (see, e.g., [18]). In this paper, we rely instead on the techniques introduced in [16] to characterize optimal controls, which do not rely on the semiconcavity of the value function and also allow for weaker regularity assumptions on the dynamics of agents. In order to obtain the classical necessary optimality conditions from Pontryagin Maximum Principle (PMP) under state constraints and few regularity assumptions, we rely on the nonsmooth PMP from [19] and make use of the technique from [20] to deal with state constraints. We also refer the interested reader to [21]-[23] for an alternative approach for dealing with other MFG models with state constraints.

Our main results are Theorem 3.6 and its Corollary 3.8 which provide the characterization of optimal controls, and Theorem 4.1, which relies on that characterization to show that equilibria of the MFG satisfy a suitable system of PDEs.

This paper is organized as follows. Section $\coprod$ presents the MFG model and the definition of equilibria and recalls the major previous results useful for this paper. We then study, in Section III the corresponding optimal control problem, providing the characterization of optimal controls under state constraints. This analysis is finally used in Section IV to show that equilibria of the mean field game satisfy a system of PDEs made of a continuity equation on the density of agents and a Hamilton-Jacobi equation on the value function of the corresponding optimal control problem.

Notation. In this paper, $d$ denotes a positive integer, the set of 
nonnegative real numbers is denoted by $\mathbb{R}_{+}, \mathbb{R}^{d}$ is endowed with its usual Euclidean norm $|\cdot|$, and $\mathbb{S}^{d-1}$ denotes the unit sphere in $\mathbb{R}^{d}$. For $R \geq 0$, we use $B_{R}$ to denote the closed ball in $\mathbb{R}^{d}$ centered at the origin and with radius $R$. The closure of a set $A \subset \mathbb{R}^{d}$ is denoted by $\bar{A}$.

Given a Polish space $X$, the set of all Borel probability measures on $X$ is denoted by $\mathscr{P}(X)$, which is always assumed to be endowed with the weak convergence of measures. When $X$ is endowed with a complete metric $d$ with respect to which $X$ is bounded, we assume that $\mathscr{P}(X)$ is endowed with the Wasserstein distance $W_{1}$ defined by $W_{1}(\mu, v)=\sup _{\phi} \int_{X} \phi(x) d(\mu-v)(x)$, where the supremum is taken over all 1-Lipschitz continuous functions $\phi: X \rightarrow \mathbb{R}$.

Given two metric spaces $X, Y$ and $M \geq 0$, we denote by $C(X, Y)$ the set of all continuous functions $f: X \rightarrow Y$, $\operatorname{Lip}(X, Y)$ the subset of $C(X, Y)$ of all Lipschitz continuous functions, and by $\operatorname{Lip}_{M}(X, Y)$ the subset of $\operatorname{Lip}(X, Y)$ of those functions whose Lipschitz constant is bounded by $M$. When $X=\mathbb{R}_{+}$, the above sets are denoted simply by $C(Y), \operatorname{Lip}(Y)$, and $\operatorname{Lip}_{M}(Y)$, respectively.

For compact $A \subset \mathbb{R}^{d}$, the space $C(A)$ is assumed to be endowed with the topology of uniform convergence on compact sets, with respect to which $C(A)$ is a Polish space. For $t \in \mathbb{R}_{+}$, we let $e_{t}: C(A) \rightarrow A$ denote the evaluation map, defined for $\gamma \in C(A)$ by $e_{t}(\gamma)=\gamma(t)$.

If $X$ and $Y$ are two metric spaces endowed with their Borel $\sigma$-algebras, $f: X \rightarrow Y$ is Borel measurable, and $\mu$ is a Borel measure in $X$, we denote the pushforward of $\mu$ through $f$ by $f_{\#} \mu$, i.e., $f_{\#} \mu$ is the Borel measure in $Y$ defined by $f_{\#} \mu(A)=$ $\mu\left(f^{-1}(A)\right)$ for every Borel subset $A$ of $Y$.

\section{DESCRIPTION OF THE MFG MODEL AND PREVIOUS RESULTS}

In this paper, we fix an open and bounded set $\Omega \subset \mathbb{R}^{d}$ and we let $\Gamma \subset \bar{\Omega}$ be a closed nonempty set. We shall always assume that $\Omega$ satisfies the following hypothesis.

(H1) There exists $D>0$ such that, for every $x, y \in \bar{\Omega}$, there exists a curve $\gamma$ included in $\bar{\Omega}$ connecting $x$ to $y$ and of length at most $D|x-y|$.

Note that $(\mathrm{H} 1)$ means that the geodesic distance in $\bar{\Omega}$ is equivalent to the usual Euclidean distance.

Let $K: \mathscr{P}(\bar{\Omega}) \times \bar{\Omega} \rightarrow \mathbb{R}_{+}$be bounded and $m_{0} \in \mathscr{P}(\bar{\Omega})$. The MFG considered in this paper, denoted by $\operatorname{MFG}\left(K, m_{0}\right)$, is described as follows. A population of agents moves in $\bar{\Omega}$ and is described at time $t \geq 0$ by a time-dependent probability measure $m_{t} \in \mathscr{P}(\bar{\Omega})$, where $m_{0}$ is the prescribed probability measure. Each agent wants to choose their trajectory in $\bar{\Omega}$ in order to reach the target set $\Gamma$ in minimal time, with the constraints that the agent must remain in $\bar{\Omega}$ at all times and that their maximal velocity at time $t$ and position $x$ is given by $K\left(m_{t}, x\right)$, i.e., the trajectory $\gamma$ of each agent solves the control system

$$
\dot{\gamma}(t)=K\left(m_{t}, \gamma(t)\right) u(t), \quad \gamma(t) \in \bar{\Omega}, u(t) \in B_{1},
$$

where $u(t)$ is the control of the agent at time $t$, chosen in order to minimize the time to reach $\Gamma$. We also assume that, once an agent reaches $\Gamma$, they stop.
Note that agents interact through the maximal velocity $K\left(m_{t}, x\right)$, which depends on the distribution of agents $m_{t}$ at time $t$. Hence, the trajectory of an agent depends on $m_{t}$ and, on the other hand, $m_{t}$ is determined by the trajectories of the agents. We are interested here in equilibrium situations, i.e., situations in which, starting from an evolution $t \mapsto m_{t}$, the optimal trajectories chosen by the agents induce an evolution of the initial distribution $m_{0}$ that coincides with $t \mapsto m_{t}$ (a more mathematically precise definition of equilibrium is provided in Definition 2.2 below).

The interaction term $K\left(m_{t}, x\right)$ can be used to model congestion phenomena in crowd motion by choosing a function $K$ such that $K\left(m_{t}, x\right)$ is small when $m_{t}$ is "large" around $x$, which means that it is harder to move on more crowded regions. For instance, $K$ may be chosen as

$$
K(\mu, x)=g\left(\int_{\bar{\Omega}} \chi(x-y) d \mu(y)\right),
$$

where $\chi$ is a convolution kernel representing the region around an agent at which they look in order to evaluate local congestion and $g$ is a decreasing function. Note that we do not assume this specific form for $K$ in this paper.

\section{A. The auxiliary optimal control problem}

Let us describe the optimal control problem solved by each agent of the game. Let $k: \mathbb{R}_{+} \times \bar{\Omega} \rightarrow \mathbb{R}_{+}$be bounded and consider the control system

$$
\dot{\gamma}(t)=k(t, \gamma(t)) u(t), \quad \gamma(t) \in \bar{\Omega}, u(t) \in B_{1},
$$

where $\gamma(t)$ is the state and $u(t)$ is the control at time $t \geq 0$. An absolutely continuous function $\gamma: \mathbb{R}_{+} \rightarrow \bar{\Omega}$ is said to be an admissible trajectory of (1) if it satisfies (1) for a.e. $t \geq 0$ for some measurable $u: \mathbb{R}_{+} \rightarrow B_{1}$, and the corresponding function $u$ is said to be the control associated with $\gamma$. The set of all admissible trajectories for (1) is denoted by $\operatorname{Adm}(k)$. For $\gamma \in \operatorname{Adm}(k)$ and $t_{0} \geq 0$, the first exit time after $t_{0}$ of $\gamma$ is the value $\tau_{\Gamma}\left(t_{0}, \gamma\right)=\inf \left\{T \geq 0 \mid \gamma\left(t_{0}+T\right) \in \Gamma\right\}$, with the convention that $\inf \varnothing=+\infty$.

We consider the optimal control problem $\operatorname{OCP}(k)$ defined as follows: given $\left(t_{0}, x_{0}\right) \in \mathbb{R}_{+} \times \bar{\Omega}$, solve

$$
\inf _{\substack{\gamma \in \operatorname{Adm}(k) \\ \gamma\left(t_{0}\right)=x_{0}}} \tau_{\Gamma}\left(t_{0}, \gamma\right)
$$

A trajectory $\gamma$ attaining the above infimum is called an optimal trajectory for $\left(k, t_{0}, x_{0}\right)$ and its associated control $u$ is called an optimal control. Note that an optimal control $u$ for $\gamma$ remains optimal if it is modified outside of the interval $\left[t_{0}, t_{0}+\tau_{\Gamma}\left(t_{0}, \gamma\right)\right]$. In order to avoid any ambiguity, we always assume, in this paper, that optimal controls are equal to 0 in the intervals $\left[0, t_{0}\right)$ and $\left(t_{0}+\tau_{\Gamma}\left(t_{0}, \gamma\right),+\infty\right)$, and in particular optimal trajectories are constant in the intervals $\left[0, t_{0}\right]$ and $\left[t_{0}+\tau_{\Gamma}\left(t_{0}, \gamma\right),+\infty\right)$. The set of all optimal trajectories for $\left(k, t_{0}, x_{0}\right)$ is denoted by $\operatorname{Opt}\left(k, t_{0}, x_{0}\right)$.

The link between $\operatorname{MFG}\left(K, m_{0}\right)$ and $\operatorname{OCP}(k)$ is that, given an evolution of agents $t \mapsto m_{t}$, each agent of the crowd solves $\operatorname{OCP}(k)$ with $k(t, x)=K\left(m_{t}, x\right)$. The optimal control problem $\operatorname{OCP}(k)$ is a minimum time problem, which is a 
classical problem in control theory for which several results are available (see, e.g., [18, Chapter 8] and [24, Chapter IV]). A classical tool in the analysis of optimal control problems is the value function $\varphi: \mathbb{R}_{+} \times \bar{\Omega} \rightarrow \mathbb{R}_{+}$, defined for $\left(t_{0}, x_{0}\right) \in$ $\mathbb{R}_{+} \times \bar{\Omega}$ by setting $\varphi\left(t_{0}, x_{0}\right)$ to be equal to the value of the infimum in (2).

We shall consider in this paper $\operatorname{OCP}(k)$ under the following assumption.

(H2) We have $k \in \operatorname{Lip}\left(\mathbb{R}_{+} \times \bar{\Omega}, \mathbb{R}_{+}\right)$and there exist positive constants $K_{\min }, K_{\max }$ such that $k(t, x) \in\left[K_{\min }, K_{\max }\right]$ for every $(t, x) \in \mathbb{R}_{+} \times \bar{\Omega}$.

We collect in the next proposition classical results on $\operatorname{OCP}(k)$ that will be of use in this paper (see, e.g., [15, Section 4]).

Proposition 2.1: Consider $\mathrm{OCP}(k)$ under hypotheses (H1) and (H2) and let $\left(t_{0}, x_{0}\right) \in \mathbb{R}_{+} \times \bar{\Omega}$.

(a) The set $\operatorname{Opt}\left(k, t_{0}, x_{0}\right)$ is nonempty.

(b) The value function $\varphi$ is Lipschitz continuous on $\mathbb{R}_{+} \times$ $\bar{\Omega}$.

(c) For every $\gamma \in \operatorname{Adm}(k)$ such that $\gamma\left(t_{0}\right)=x_{0}$, we have, for every $h \geq 0$,

$$
\varphi\left(t_{0}+h, \gamma\left(t_{0}+h\right)\right)+h \geq \varphi\left(t_{0}, x_{0}\right),
$$

with equality if $\gamma \in \operatorname{Opt}\left(k, t_{0}, x_{0}\right)$ and $h \in\left[0, \tau_{\Gamma}\left(t_{0}, \gamma\right)\right]$. Conversely, if $\gamma \in \operatorname{Adm}(k)$ satisfies $\gamma\left(t_{0}\right)=x_{0}$, if $\gamma$ is constant on $\left[0, t_{0}\right]$ and on $\left[t_{0}+\tau_{\Gamma}\left(t_{0}, \gamma\right),+\infty\right)$, and if equality holds in (3) for every $h \in\left[0, \tau_{\Gamma}\left(t_{0}, \gamma\right)\right]$, then $\gamma \in \operatorname{Opt}\left(k, t_{0}, x_{0}\right)$.

(d) The value function $\varphi$ satisfies the Hamilton-Jacobi equation

$$
-\partial_{t} \varphi(t, x)+|\nabla \varphi(t, x)| k(t, x)-1=0
$$

in the following sense: $\varphi$ is a viscosity subsolution of (4) in $\mathbb{R}_{+} \times(\Omega \backslash \Gamma)$, a viscosity supersolution of (4) in $\mathbb{R}_{+} \times(\bar{\Omega} \backslash \Gamma)$, and satisfies $\varphi(t, x)=0$ for every $(t, x) \in$ $\mathbb{R}_{+} \times \Gamma$.

(e) If $\gamma \in \operatorname{Opt}\left(k, t_{0}, x_{0}\right), t \in\left[t_{0}, t_{0}+\varphi\left(t_{0}, x_{0}\right)\right), \gamma(t) \in \Omega \backslash \Gamma$, and $\varphi$ is differentiable at $(t, \gamma(t))$, then $|\nabla \varphi(t, \gamma(t))| \neq 0$ and

$$
\dot{\gamma}(t)=-k(t, \gamma(t)) \frac{\nabla \varphi(t, \gamma(t))}{|\nabla \varphi(t, \gamma(t))|} .
$$

\section{B. Lagrangian equilibria and their existence}

In this paper, we study $\operatorname{MFG}\left(K, m_{0}\right)$ in a Lagrangian setting, in which the evolution of agents is described by a measure $Q \in \mathscr{P}(C(\bar{\Omega}))$ in the space of all continuous trajectories $C(\bar{\Omega})$. This classical approach in optimal transport has become widely used in the analysis of MFGs with deterministic trajectories in recent years (see, e.g., [13], [15], [21], [25], [26]). Note that the distribution $m_{t}$ of agents at time $t \geq 0$ can be retrieved from $Q$ using the evaluation map $e_{t}$ by $m_{t}=e_{t \#} Q$. The definition of an equilibrium of $\operatorname{MFG}\left(K, m_{0}\right)$ is formulated in the Lagrangian setting as follows.

Definition 2.2: Consider $\operatorname{MFG}\left(K, m_{0}\right)$. A measure $Q \in$ $\mathscr{P}(C(\bar{\Omega}))$ is called a Lagrangian equilibrium (or simply equilibrium) of $\operatorname{MFG}\left(K, m_{0}\right)$ if $e_{0 \#} Q=m_{0}$ and $Q$-almost every $\gamma \in C(\bar{\Omega})$ is an optimal curve for $(k, 0, \gamma(0))$, where $k: \mathbb{R}_{+} \times \bar{\Omega} \rightarrow \mathbb{R}_{+}$is defined by $k(t, x)=K\left(e_{t \#} Q, x\right)$.

The next assumption is the counterpart of (H2) for $\operatorname{MFG}\left(K, m_{0}\right)$.

(H3) We have $K \in \operatorname{Lip}\left(\mathscr{P}(\bar{\Omega}) \times \bar{\Omega}, \mathbb{R}_{+}\right)$and there exist positive constants $K_{\min }, K_{\max }$ such that $K(\mu, x) \in$ $\left[K_{\min }, K_{\max }\right]$ for every $(\mu, x) \in \mathscr{P}(\bar{\Omega}) \times \bar{\Omega}$.

We recall in the next theorem the main result of [15] concerning existence of equilibria.

Theorem 2.3: Consider $\operatorname{MFG}\left(K, m_{0}\right)$ under assumptions (H1) and (H3) Then there exists an equilibrium $Q \in$ $\mathscr{P}(C(\bar{\Omega}))$ for $\operatorname{MFG}\left(K, m_{0}\right)$.

\section{FURTHER PROPERTIES OF THE OPTIMAL CONTROL PROBLEM}

We provide in this section further properties of $\mathrm{OCP}(k)$ with the aim of providing a characterization of optimal controls. For that purpose, we assume the following additional hypothesis on $\Omega$.

(H4) The boundary $\partial \Omega$ is a compact $C^{1,1}$ manifold.

We will denote in the sequel by $d^{ \pm}$the signed distance to $\partial \Omega$, defined by $d^{ \pm}(x)=d(x, \Omega)-d\left(x, \mathbb{R}^{d} \backslash \Omega\right)$, where $d(x, A)=\inf _{y \in A}|x-y|$ for $A \subset \mathbb{R}^{d}$. Recall that, under assumption (H4), $d^{ \pm}$is $C^{1,1}$ in a neighborhood of $\partial \Omega$, its gradient has unit norm, and $\nabla d^{ \pm}$is a Lipschitz continuous function extending the exterior normal vector field of $\Omega$ to a neighborhood of $\partial \Omega$ (see, e.g., [27]).

\section{A. Consequences of Pontryagin Maximum Principle}

In order to obtain additional properties of optimal trajectories, we apply Pontryagin Maximum Principle to a modified optimal control problem without state constraints, following the techniques from [20]. Assume that $k$ satisfies (H2) and is extended to a Lipschitz continuous function defined on $\mathbb{R}_{+} \times \mathbb{R}^{d}$. We also assume, with no loss of generality, that the extension of $k$ is $C^{1}$ on $\mathbb{R}_{+} \times\left(\mathbb{R}^{d} \backslash \bar{\Omega}\right)$. For $\varepsilon>0$, define $k_{\varepsilon}: \mathbb{R}_{+} \times \mathbb{R}^{d} \rightarrow \mathbb{R}_{+}$by

$$
k_{\varepsilon}(t, x)=k(t, x)\left(1-\frac{1}{\varepsilon} d(x, \Omega)\right)_{+},
$$

where $a_{+}$is defined by $a_{+}=\max (0, a)$ for $a \in \mathbb{R}$. Consider the control system

$$
\dot{\gamma}_{\varepsilon}(t)=k_{\varepsilon}\left(t, \gamma_{\varepsilon}(t)\right) u_{\varepsilon}(t), \quad \gamma_{\varepsilon}(t) \in \mathbb{R}^{d}, u_{\varepsilon}(t) \in B_{1}
$$

and the optimal control problem $\operatorname{OCP}_{\varepsilon}\left(k_{\varepsilon}\right)$ of, given $\left(t_{0}, x_{0}\right) \in$ $\mathbb{R}_{+} \times \mathbb{R}^{d}$, finding a measurable control $u_{\varepsilon}$ such that the corresponding trajectory $\gamma_{\varepsilon}$ solving (6) reaches $\Gamma$ in minimal time. The next lemma states the main consequences of Pontryagin Maximum Principle when applied to $\mathrm{OCP}_{\varepsilon}\left(k_{\varepsilon}\right)$.

Lemma 3.1: Consider $\mathrm{OCP}_{\varepsilon}\left(k_{\varepsilon}\right)$ under assumptions (H1), $(\mathrm{H} 2)$, and (H4) and with $k_{\varepsilon}$ defined by (5). Let $\left(t_{0}, x_{0}\right) \in$ $\mathbb{R}_{+} \times \bar{\Omega}, \gamma_{\varepsilon}$ be an optimal trajectory for $\operatorname{OCP}_{\varepsilon}\left(k_{\varepsilon}\right), T_{\varepsilon}$ be the first exit time of $\gamma_{\varepsilon}$, and $u_{\varepsilon}:\left[t_{0}, t_{0}+T_{\varepsilon}\right] \rightarrow B_{1}$ be an optimal control associated with $\gamma_{\varepsilon}$. Then $d\left(\gamma_{\varepsilon}(t), \Omega\right)<\varepsilon$ for every $t \in\left[t_{0}, t_{0}+T_{\varepsilon}\right]$ and there exist $\lambda_{\varepsilon} \in\{0,1\}$ and absolutely 
continuous functions $p_{\varepsilon}:\left[t_{0}, t_{0}+T_{\varepsilon}\right] \rightarrow \mathbb{R}^{d}$ and $q_{\varepsilon}:\left[t_{0}, t_{0}+\right.$ $\left.T_{\varepsilon}\right] \rightarrow \mathbb{R}$ such that, for a.e. $t \in\left[t_{0}, t_{0}+T_{\varepsilon}\right]$,

$$
\begin{aligned}
\dot{q}_{\varepsilon}(t) & \in\left|p_{\varepsilon}(t)\right| \pi_{1} \partial^{C} k_{\varepsilon}\left(t, \gamma_{\varepsilon}(t)\right), \\
-\dot{p}_{\varepsilon}(t) & \in\left|p_{\varepsilon}(t)\right| \pi_{2} \partial^{C} k_{\varepsilon}\left(t, \gamma_{\varepsilon}(t)\right), \\
q_{\varepsilon}(t) & =\left|p_{\varepsilon}(t)\right| k_{\varepsilon}\left(t, \gamma_{\varepsilon}(t)\right)-\lambda_{\varepsilon}, \\
\max _{w \in B_{1}} p_{\varepsilon}(t) \cdot w & =p_{\varepsilon}(t) \cdot u_{\varepsilon}(t), \\
q_{\varepsilon}\left(t_{0}+T_{\varepsilon}\right) & =0, \\
\lambda_{\varepsilon}+\max _{t \in\left[t_{0}, t_{0}+T_{\varepsilon}\right]}\left|p_{\varepsilon}(t)\right| & >0,
\end{aligned}
$$

where $\partial^{C}$ denotes Clarke's gradient (see [19] for its definition) and $\pi_{1}, \pi_{2}$ are the projections onto the first and second factors of the product $\mathbb{R} \times \mathbb{R}^{d}$, respectively.

The proof of Lemma 3.1 is standard and can be carried out by showing first that $d\left(\gamma_{\varepsilon}(t), \Omega\right)<\varepsilon$ for every $t \in\left[t_{0}, t_{0}+T_{\varepsilon}\right]$, which holds since, otherwise, $\gamma_{\varepsilon}$ would belong, for some time, to a region outside of $\bar{\Omega}$ where $k_{\varepsilon}$ is identically zero, and hence $\gamma_{\varepsilon}$ would be constant, contradicting its optimality. With this fact, we can apply [19, Theorem 5.2.3] to the autonomous augmented system $\frac{d}{d t}\left(t, \gamma_{\varepsilon}(t)\right)=\left(1, k_{\varepsilon}\left(t, \gamma_{\varepsilon}(t)\right) u_{\varepsilon}(t)\right)$ and deduce (7) from its conclusions.

As a consequence of Lemma 3.1 we obtain the following properties of optimal controls for $\mathrm{OCP}_{\varepsilon}\left(k_{\varepsilon}\right)$.

Lemma 3.2: Under the assumption and notations of Lemma 3.1, for every $t \in\left[t_{0}, t_{0}+T_{\varepsilon}\right]$, we have $\left|p_{\varepsilon}(t)\right| \neq 0$ and $u_{\varepsilon}(t)=\frac{p_{\varepsilon}(t)}{p_{\varepsilon}(t)}$. As a consequence, $u_{\varepsilon}$ is Lipschitz continuous and $\gamma_{\varepsilon}$ is $C^{1,1}$, and the Lipschitz constant of $u_{\varepsilon}$ depends only on $\varepsilon, K_{\max }$, and the Lipschitz constant of $k$.

Proof: Let $L$ be the Lipschitz constant of $k$. From the definition of $k_{\varepsilon}$ and standard properties of Clarke's gradient (see, e.g., [19, Proposition 2.1.2]), we have that $|\zeta| \leq L+$ $\frac{K_{\max }}{\varepsilon}$ for every $(t, x) \in \mathbb{R}_{+} \times \mathbb{R}^{d}$ and $\zeta \in \pi_{2} \partial^{C} k_{\varepsilon}(t, x)$. Hence, integrating (7b), we deduce that, for every $t, t_{1} \in\left[t_{0}, t_{0}+T_{\varepsilon}\right]$,

$$
\left|p_{\varepsilon}(t)\right| \leq\left|p_{\varepsilon}\left(t_{1}\right)\right|+\left(L+\frac{K_{\max }}{\varepsilon}\right) \int_{\min \left\{t, t_{1}\right\}}^{\max \left\{t, t_{1}\right\}}\left|p_{\varepsilon}(s)\right| d s .
$$

Hence, by Grönwall's inequality, for every $t, t_{1} \in\left[t_{0}, t_{0}+T_{\varepsilon}\right]$,

$$
\left|p_{\varepsilon}(t)\right| \leq\left|p_{\varepsilon}\left(t_{1}\right)\right| e^{\left(L+\frac{K_{m a x}}{\varepsilon}\right)\left|t-t_{1}\right|} .
$$

If there exists $t_{1} \in\left[t_{0}, t_{0}+T_{\varepsilon}\right]$ such that $p_{\varepsilon}\left(t_{1}\right)=0$, then $p_{\varepsilon}(t)=0$ for every $t \in\left[t_{0}, t_{0}+T_{\varepsilon}\right]$. Thus, by $(\overline{7 c}), q_{\varepsilon}(t)=-\lambda_{\varepsilon}$ for every $t \in\left[t_{0}, t_{0}+T_{\varepsilon}\right]$, and since $q_{\varepsilon}\left(t_{0}+T_{\mathcal{E}}\right)=0$ by $(7 \mathrm{e})$, it follows that $\lambda_{\varepsilon}=0$, which contradicts $(7 \mathrm{f})$, establishing thus that $\left|p_{\varepsilon}(t)\right| \neq 0$ for every $t \in\left[t_{0}, t_{0}+T_{\varepsilon}\right]$.

Thanks to this fact, one deduces immediately from (7d) that $u_{\varepsilon}(t)=\frac{p_{\varepsilon}(t)}{\left|p_{\varepsilon}(t)\right|}$. Denoting by $b_{\varepsilon}:\left[t_{0}, t_{0}+T_{\varepsilon}\right] \rightarrow \mathbb{R}^{d}$ a measurable function such that $-\dot{p}_{\varepsilon}(t)=\left|p_{\varepsilon}(t)\right| b_{\varepsilon}(t)$ for a.e. $t \in\left[t_{0}, t_{0}+T_{\varepsilon}\right]$, we deduce that, for a.e. $t \in\left[t_{0}, t_{0}+T_{\varepsilon}\right]$,

$$
\dot{u}_{\varepsilon}(t)=-b_{\varepsilon}(t)+\left(u_{\varepsilon}(t) \cdot b_{\varepsilon}(t)\right) u_{\varepsilon}(t) .
$$

Since $b_{\varepsilon}(t) \in \pi_{2} \partial^{C} k_{\varepsilon}\left(t, \gamma_{\varepsilon}(t)\right)$ for a.e. $t \in\left[t_{0}, t_{0}+T_{\varepsilon}\right]$, we conclude that $\left|\dot{u}_{\varepsilon}(t)\right| \leq L+\frac{K_{\max }}{\varepsilon}$, showing that $u$ is Lipschitz continuous, as required.
Similarly to [20], we now establish the main link between $\mathrm{OCP}(k)$ and $\mathrm{OCP}_{\varepsilon}\left(k_{\varepsilon}\right)$.

Proposition 3.3: Consider $\mathrm{OCP}(k)$ under the assumptions (H1), (H2), and (H4), as well as the problem $\mathrm{OCP}_{\varepsilon}\left(k_{\varepsilon}\right)$ with $k_{\varepsilon}$ defined by (5). There exists $\varepsilon_{0}>0$ such that, for every $\varepsilon \in\left(0, \varepsilon_{0}\right)$ and $\left(t_{0}, x_{0}\right) \in \mathbb{R} \times \bar{\Omega}$, the following properties hold.

(a) If $\gamma_{\varepsilon}$ is an optimal trajectory for $\mathrm{OCP}_{\varepsilon}\left(k_{\varepsilon}\right)$ starting from $\left(t_{0}, x_{0}\right)$, then $\gamma_{\varepsilon}(t) \in \bar{\Omega}$ for every $t \geq 0$.

(b) If $\gamma \in \operatorname{Opt}\left(k, t_{0}, x_{0}\right)$, then $\gamma$ is an optimal trajectory for $\mathrm{OCP}_{\varepsilon}\left(k_{\varepsilon}\right)$.

As a consequence, if $\gamma \in \operatorname{Opt}\left(k, t_{0}, x_{0}\right)$ and $u$ is its associated optimal control, then $\gamma$ is $C^{1,1}$ and $u$ is Lipschitz continuous on $\left[t_{0}, t_{0}+\tau_{\Gamma}\left(t_{0}, \gamma\right)\right]$, and the Lipschitz constant of $u$ depends only on $\varepsilon_{0}, K_{\max }$, and the Lipschitz constant of $k$.

Proof: To prove (a), let $T_{\varepsilon}$ be the first exit time of $\gamma_{\varepsilon}$ and assume, to obtain a contradiction, that there exist $a, b \in\left[t_{0}, t_{0}+T_{\varepsilon}\right]$ such that $a<b, \gamma_{\varepsilon}(t) \notin \bar{\Omega}$ for $t \in(a, b)$, and $\gamma_{\varepsilon}(t) \in \partial \Omega$ for $t \in\{a, b\}$ (recall that $\Gamma \subset \bar{\Omega}$ and $x_{0} \in \bar{\Omega}$, so $\gamma_{\varepsilon}$ starts and ends its movement in $\bar{\Omega})$. The map $t \mapsto d^{ \pm}\left(\gamma_{\varepsilon}(t)\right)$ is differentiable in a neighborhood of $[a, b]$, strictly positive for $t \in(a, b)$, and equal to 0 for $t \in\{a, b\}$, and thus its derivative is nonnegative at $a$ and nonpositive at $b$, i.e.,

$$
\dot{\gamma}_{\varepsilon}(a) \cdot \nabla d^{ \pm}\left(\gamma_{\varepsilon}(a)\right) \geq 0, \quad \dot{\gamma}_{\varepsilon}(b) \cdot \nabla d^{ \pm}\left(\gamma_{\varepsilon}(b)\right) \leq 0 .
$$

Since $\dot{\gamma}_{\varepsilon}(t)=k_{\varepsilon}\left(t, \gamma_{\varepsilon}(t)\right) \frac{p_{\varepsilon}(t)}{p_{\varepsilon}(t) \mid}$ and $\frac{k_{\varepsilon}\left(t, \gamma_{\varepsilon}(t)\right)}{\left|p_{\varepsilon}(t)\right|}>0$ for every $t \in\left[t_{0}, t_{0}+T_{\varepsilon}\right]$, we have

$$
p_{\varepsilon}(a) \cdot \nabla d^{ \pm}\left(\gamma_{\varepsilon}(a)\right) \geq 0, \quad p_{\varepsilon}(b) \cdot \nabla d^{ \pm}\left(\gamma_{\varepsilon}(b)\right) \leq 0 .
$$

Consider the map $\alpha: t \mapsto p_{\varepsilon}(t) \cdot \nabla d^{ \pm}\left(\gamma_{\varepsilon}(t)\right)$. Since $d^{ \pm}$is $C^{1,1}$ in a neighborhood of $\partial \Omega$ and $d\left(\gamma_{\varepsilon}(t), \Omega\right) \leq \varepsilon$ for every $t \in\left[t_{0}, t_{0}+T_{\varepsilon}\right]$ by Lemma 3.1 , if $\varepsilon_{0}>0$ is small enough, we deduce that $\gamma_{\varepsilon}(t)$ belongs to the neighborhood at which $d^{ \pm}$ is $C^{1,1}$ for every $t \in(a, b)$. Thus $\alpha$ is absolutely continuous on $[a, b]$ and, recalling that $k$ is $C^{1}$ on $\mathbb{R}_{+} \times\left(\mathbb{R}^{d} \backslash \bar{\Omega}\right)$ and using (7b), we have, for $t \in(a, b)$,

$$
\begin{aligned}
& \dot{\alpha}(t)=\dot{p}_{\varepsilon}(t) \cdot \nabla d^{ \pm}\left(\gamma_{\varepsilon}(t)\right)+p_{\varepsilon}(t) \cdot \frac{d\left[\nabla d^{ \pm} \circ \gamma_{\varepsilon}\right]}{d t}(t) \\
& =-\left|p_{\varepsilon}(t)\right|\left(1-\frac{1}{\varepsilon} d\left(\gamma_{\varepsilon}(t), \Omega\right)\right)_{+} \nabla_{x} k\left(t, \gamma_{\varepsilon}(t)\right) \cdot \nabla d^{ \pm}\left(\gamma_{\varepsilon}(t)\right) \\
& +\left|p_{\varepsilon}(t)\right| \frac{1}{\varepsilon} k\left(t, \gamma_{\varepsilon}(t)\right)\left|\nabla d^{ \pm}\left(\gamma_{\varepsilon}(t)\right)\right|^{2}+p_{\varepsilon}(t) \cdot \frac{d\left[\nabla d^{ \pm} \circ \gamma_{\varepsilon}\right]}{d t}(t) \\
& \geq\left|p_{\varepsilon}(t)\right|\left[-L+\frac{K_{\min }}{\varepsilon}-L K_{\max }\right]
\end{aligned}
$$

where $L$ is an upper bound on the Lipschitz constants of $d^{ \pm}$ and $k$. Up to decreasing $\varepsilon_{0}$, we have $-L+\frac{K_{\min }}{\varepsilon}-L K_{\max }>0$ for every $\varepsilon \in\left(0, \varepsilon_{0}\right)$, and hence $\dot{\alpha}(t)>0$ for $t \in(a, b)$, which contradicts (8). This contradiction establishes (a).

To establish (b), let $\gamma_{\varepsilon}$ be an optimal trajectory for $\mathrm{OCP}_{\varepsilon}\left(k_{\varepsilon}\right)$ starting at $\left(t_{0}, x_{0}\right)$ and denote by $T_{\varepsilon}$ its first exit time after $t_{0}$. Since $\gamma \in \operatorname{Opt}\left(k, t_{0}, x_{0}\right), \gamma$ is admissible for $\mathrm{OCP}_{\varepsilon}\left(k_{\varepsilon}\right)$, and thus $\tau_{\Gamma}\left(t_{0}, \gamma\right) \geq T_{\varepsilon}$. On the other hand, by (a), we have $\gamma_{\varepsilon} \in \operatorname{Adm}(k)$, and thus $T_{\varepsilon} \leq \tau_{\Gamma}\left(t_{0}, \gamma\right)$. Thus $\tau_{\Gamma}\left(t_{0}, \gamma\right)=T_{\mathcal{\varepsilon}}$, concluding the proof. 


\section{B. Boundary condition of the Hamilton-Jacobi equation}

Having established in particular in Proposition 3.3 that optimal controls for $\mathrm{OCP}(k)$ are Lipschitz continuous, we are now able to deduce a boundary condition for the HamiltonJacobi equation (4).

Proposition 3.4: Consider $\mathrm{OCP}(k)$ under the assumptions (H1), (H2) and (H4), and let $\varphi$ be its value function and $\mathbf{n}$ be the exterior normal of $\Omega$. Then $\varphi$ satisfies $\nabla \varphi(t, x) \cdot \mathbf{n}(x) \geq 0$ for $(t, x) \in \mathbb{R}_{+} \times(\partial \Omega \backslash \Gamma)$ in the viscosity supersolution sense.

Proof: Let $\left(t_{0}, x_{0}\right) \in \mathbb{R}_{+} \times(\partial \Omega \backslash \Gamma)$ and $\xi$ be a smooth function defined on a neighborhood $V$ of $\left(t_{0}, x_{0}\right)$ in $\mathbb{R}_{+} \times \bar{\Omega}$ such that $\xi\left(t_{0}, x_{0}\right)=\varphi\left(t_{0}, x_{0}\right)$ and $\xi(t, x) \leq \varphi(t, x)$ for $(t, x) \in$ $V$. Assume, to obtain a contradiction, that $\nabla \xi\left(t_{0}, x_{0}\right) \cdot \mathbf{n}\left(x_{0}\right)<$ 0 . Let $\gamma \in \operatorname{Opt}\left(k, t_{0}, x_{0}\right)$, denote by $u$ its associated optimal control, and define $\tilde{\gamma}:\left[t_{0}-\varepsilon,+\infty\right) \rightarrow \bar{\Omega}$ for $\varepsilon>0$ small enough by $\tilde{\gamma}(t)=\gamma(t)$ for $t \geq t_{0}$ and as the solution of $\dot{\tilde{\gamma}}(t)=$ $-k(t, \tilde{\gamma}(t)) \frac{\nabla \xi\left(t_{0}, x_{0}\right)}{\left|\nabla \xi\left(t_{0}, x_{0}\right)\right|}$ for $t \in\left[t_{0}-\varepsilon, t_{0}\right]$ with final condition $\tilde{\gamma}\left(t_{0}\right)=x_{0}$ (we extend $k$ to negative times in a Lipschitz manner if needed). Applying Proposition 2.1 (c) to $\tilde{\gamma}$, we get that $\varphi\left(t_{0}, x_{0}\right) \geq \varphi\left(t_{0}-h, \tilde{\gamma}\left(t_{0}-h\right)\right)-h$ for every $h \in[0, \varepsilon]$, and thus $\xi\left(t_{0}, x_{0}\right) \geq \xi\left(t_{0}-h, \tilde{\gamma}\left(t_{0}-h\right)\right)-h$. Since $\xi\left(t_{0}-h, \tilde{\gamma}\left(t_{0}-\right.\right.$ $h))=\xi\left(t_{0}, x_{0}\right)-h \partial_{t} \xi\left(t_{0}, x_{0}\right)-h \dot{\tilde{\gamma}}\left(t_{0}^{-}\right) \cdot \nabla \xi\left(t_{0}, x_{0}\right)+o(h)$, we deduce that

$$
-\partial_{t} \xi\left(t_{0}, x_{0}\right)+k\left(t_{0}, x_{0}\right)\left|\nabla \xi\left(t_{0}, x_{0}\right)\right|-1 \leq 0 .
$$

Since $\gamma \in \operatorname{Opt}\left(k, t_{0}, x_{0}\right)$, we have, by Proposition 2.1] (c). that $\varphi\left(t_{0}, x_{0}\right)=\varphi\left(t_{0}+h, \gamma\left(t_{0}+h\right)\right)+h$ for $h \geq 0$ small enough, and thus $\xi\left(t_{0}, x_{0}\right) \geq \xi\left(t_{0}+h, \tilde{\gamma}\left(t_{0}+h\right)\right)+h$. Performing the first order expansion of $\xi\left(t_{0}+h, \gamma\left(t_{0}+h\right)\right)$ on $h$ as before and using the fact that $\gamma$ satisfies (1), we deduce that $\partial_{t} \xi\left(t_{0}, x_{0}\right)+k\left(t_{0}, x_{0}\right) \nabla \xi\left(t_{0}, x_{0}\right) \cdot u\left(t_{0}\right)+1 \leq 0$. Adding with (9), we deduce that $\nabla \xi\left(t_{0}, x_{0}\right) \cdot u\left(t_{0}\right)+\left|\nabla \xi\left(t_{0}, x_{0}\right)\right| \leq 0$ and, since $u\left(t_{0}\right) \in B_{1}$, this implies that $u\left(t_{0}\right)=-\frac{\nabla \xi\left(t_{0}, x_{0}\right)}{\left|\nabla \xi\left(t_{0}, x_{0}\right)\right|}$. Since $\nabla \xi\left(t_{0}, x_{0}\right) \cdot \mathbf{n}\left(x_{0}\right)<0$, this would imply that $\gamma$ leaves $\bar{\Omega}$ at some $t_{0}+h$ for $h>0$ small enough, contradicting the fact that $\gamma \in \operatorname{Opt}\left(k, t_{0}, x_{0}\right)$. This contradiction establishes that $\nabla \xi\left(t_{0}, x_{0}\right) \cdot \mathbf{n}\left(x_{0}\right) \geq 0$, as required.

\section{Characterization of optimal controls}

Using Propositions 3.3 and 3.4 , we are now in position to characterize optimal controls of $\operatorname{OCP}(k)$. We start by introducing the two main objects that we will use in our characterization.

Definition 3.5: Consider $\mathrm{OCP}(k)$ under assumptions $(\mathrm{H} 1)$ $(\mathrm{H} 2)$, and (H4) Let $\varphi$ be its value function and take $\left(t_{0}, x_{0}\right) \in$ $\mathbb{R}_{+} \times \bar{\Omega}$.

(a) We define the set $\mathscr{U}\left(t_{0}, x_{0}\right)$ of optimal directions at $\left(t_{0}, x_{0}\right)$ as the set of $u_{0} \in \mathbb{S}^{d-1}$ for which there exists $\gamma \in$ $\operatorname{Opt}\left(k, t_{0}, x_{0}\right)$ such that the optimal control $u$ associated with $\gamma$ satisfies $u\left(t_{0}\right)=u_{0}$.

(b) We define the set $\mathscr{W}\left(t_{0}, x_{0}\right)$ of directions of maximal descent of $\varphi$ at $\left(t_{0}, x_{0}\right)$ as the set of $u_{0} \in \mathbb{S}^{d-1}$ such that

$$
\lim _{h \rightarrow 0^{+}} \frac{\varphi\left(t_{0}+h, x_{0}+h k\left(t_{0}, x_{0}\right) u_{0}\right)-\varphi\left(t_{0}, x_{0}\right)}{h}=-1 .
$$

Note that $\mathscr{U}\left(t_{0}, x_{0}\right) \neq \varnothing$ for $x_{0} \in \bar{\Omega} \backslash \Gamma$ and, by Proposition 2.1 (c), the quantity on the left-hand side of (10) whose limit is being computed is greater than or equal to $-1+o(1)$ as $h \rightarrow 0^{+}$. The main result of this section is the following.

Theorem 3.6: Consider $\operatorname{OCP}(k)$ and its value function $\varphi$ under (H1), (H2), and (H4) and let $\left(t_{0}, x_{0}\right) \in \mathbb{R}_{+} \times \bar{\Omega}$.

(a) If $\varphi$ is differentiable at $\left(t_{0}, x_{0}\right)$, then $\mathscr{W}\left(t_{0}, x_{0}\right)=$ $\left\{-\frac{\nabla \varphi\left(t_{0}, x_{0}\right)}{\left|\nabla \varphi\left(t_{0}, x_{0}\right)\right|}\right\}$.

(b) For every $\gamma \in \operatorname{Opt}\left(k, t_{0}, x_{0}\right)$ and $t \in\left(t_{0}, t_{0}+\varphi\left(t_{0}, x_{0}\right)\right)$, $\mathscr{U}(t, \gamma(t))$ contains exactly one element.

(c) We have $\mathscr{U}\left(t_{0}, x_{0}\right)=\mathscr{W}\left(t_{0}, x_{0}\right)$.

Proof: Assertion (a) follows easily from (10) by using Proposition 2.1[d) and (e) (see also [16, Proposition 4.13] for a proof in the case with no state constraints). Assertion (b) follows from the fact that optimal controls are Lipschitz continuous (Proposition 3.3 and its proof is very similar to that of [15, Proposition 4.7]. As for assertion (c) its proof is very similar to that of [16, Theorem 4.14] and we sketch it here for completeness. First, note that it suffices to consider the case $x_{0} \in \bar{\Omega} \backslash \Gamma$ since both sets are empty if $x_{0} \in \Gamma$. The inclusion $\mathscr{U}\left(t_{0}, x_{0}\right) \subset \mathscr{W}\left(t_{0}, x_{0}\right)$ can be obtained by applying Proposition 2.1 (c) and taking the limit as $h \rightarrow 0^{+}$in (10). For the converse inclusion, let $u_{0} \in \mathscr{W}\left(t_{0}, x_{0}\right)$ and note that, if $x_{0} \in \partial \Omega \backslash \Gamma$, then necessarily $u_{0}$ points towards the inside of $\Omega$. Let $\gamma_{0}$ be the solution of (1) starting from $\left(t_{0}, x_{0}\right)$ and with constant control $u_{0}$, defined in $\left[t_{0}, t_{0}+h\right]$ for some $h>0$ small enough, define $t_{1}=t_{0}+h$ and $x_{1}=\gamma_{0}\left(t_{0}+h\right)$, and let $\gamma_{1} \in \operatorname{Opt}\left(k, t_{1}, x_{1}\right)$. The conclusion follows by letting $h \rightarrow 0^{+}$ if one assumes that the optimal control $u_{1}$ associated with $\gamma_{1}$ satisfies $u_{1}\left(t_{1}\right) \rightarrow u_{0}$ as $h \rightarrow 0^{+}$, using the fact that limits of optimal trajectories are also optimal.

We prove by contradiction that we necessarily have $u_{1}\left(t_{1}\right) \rightarrow u_{0}$ as $h \rightarrow 0^{+}$. Indeed, assume that this is not the case, let $\bar{\gamma}_{1}$ be the solution of (1) starting from $\left(t_{1}, x_{1}\right)$ and with constant control $u_{1}\left(t_{1}\right)$, and define $t_{2}=t_{1}+h$, $x_{2}=\gamma_{1}\left(t_{2}\right)$, and $\bar{x}_{2}=\bar{\gamma}_{1}\left(t_{2}\right)$. Define also $\gamma_{2}$ as the solution of (1) starting from $\left(t_{0}, x_{0}\right)$ and with constant control $\frac{\bar{x}_{2}-x_{0}}{\left|\bar{x}_{2}-x_{0}\right|}, \tau$ be the time at which $\gamma_{2}$ arrives at $\bar{x}_{2}$, and $\gamma_{3}$ be the solution of (1) starting from $\left(\tau, \bar{x}_{2}\right)$ and with constant control $\frac{x_{2}-\bar{x}_{2}}{\left|x_{2}-\bar{x}_{2}\right|}$ (see Figure 1 for an illustration of these constructions). Note that, since $u_{0}$ points towards the inside of $\Omega$, all points and trajectories in this construction remain in $\bar{\Omega}$ for $h$ small enough.

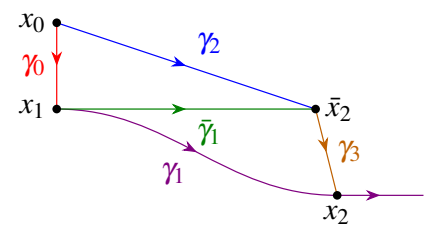

Fig. 1. Illustration of the constructions used in the proof of Theorem 3.([) (adapted from [16]).

Since the angle between $\gamma_{0}$ and $\bar{\gamma}_{1}$ at $x_{1}$ is different from $\pi$ as $h \rightarrow 0^{+}$, one can prove that there exists $\rho<1$ such that the time $\tau-t_{0}$ that $\gamma_{2}$ takes to go from $x_{0}$ to $\bar{x}_{2}$ is at most 
$2 \rho h+O\left(h^{2}\right)$. On the other hand, since $\gamma_{1}$ and $\bar{\gamma}_{1}$ are tangent at $x_{1}$, the time that $\gamma_{3}$ takes to go from $\bar{x}_{2}$ to $x_{2}$ is at most $O\left(h^{2}\right)$. We have thus constructed two trajectories to go from $x_{0}$ to $x_{2}$ : one obtained as the concatenation of $\gamma_{0}$ and $\gamma_{1}$, which takes a time $2 h$, and another obtained as the concatenation of $\gamma_{2}$ and $\gamma_{3}$, which takes a time $2 \rho h+O\left(h^{2}\right)<2 h$. By applying Proposition 2.1 (c) to both trajectories, letting $h \rightarrow 0^{+}$, and using [15, Proposition 4.4], one gets the conclusion that $\rho \geq$ 1 , yielding the desired contradiction.

Motivated by Theorem 3.4[a) we provide the following definition.

Definition 3.7: Consider $\mathrm{OCP}(k)$ under assumptions $(\mathrm{H} 1)$ $(\mathrm{H} 2)$, and (H4), let $\varphi$ be its value function, and take $\left(t_{0}, x_{0}\right) \in$ $\mathbb{R}_{+} \times \bar{\Omega}$. If $\mathscr{W}\left(t_{0}, x_{0}\right)$ contains exactly one element, we denote this element by $-\widehat{\nabla \varphi}\left(t_{0}, x_{0}\right)$, and call it the normalized gradient of $\varphi$ at $\left(t_{0}, x_{0}\right)$.

As a consequence of Theorem 3.6, we obtain the following characterization of optimal trajectories.

Corollary 3.8: Consider $\mathrm{OCP}(k)$ under assumptions (H1), $(\mathrm{H} 2)$, and (H4), let $\varphi$ be its value function, and take $\left(t_{0}, x_{0}\right) \in$ $\mathbb{R}_{+} \times \bar{\Omega}$ and $\gamma \in \operatorname{Opt}\left(k, t_{0}, x_{0}\right)$. Then, for every $t \in\left(t_{0}, t_{0}+\right.$ $\left.\varphi\left(t_{0}, x_{0}\right)\right), \varphi$ admits a normalized gradient at $(t, \gamma(t))$ and $\dot{\gamma}(t)=-k(t, \gamma(t)) \widehat{\nabla \varphi}(t, \gamma(t))$.

We also have the following result on the normalized gradient, whose proof is very similar to that of [16, Proposition 4.17] (see also [15, Proposition 4.9]).

Proposition 3.9: Consider $\mathrm{OCP}(k)$ and its value function $\varphi$ under assumptions (H1) (H2), and (H4), Then $\widehat{\nabla \varphi}$ is continuous on its domain of definition.

\section{THE MFG SysteM}

Following the results on $\mathrm{OCP}(k)$ and using the relation between $\operatorname{MFG}\left(K, m_{0}\right)$ and $\operatorname{OCP}(k)$, we are now ready to obtain, as a consequence of Proposition 2.1(d), Proposition 3.4, Corollary 3.8, and Proposition 3.9, that equilibria of $\operatorname{MFG}\left(K, m_{0}\right)$ satisfy a system of PDEs.

Theorem 4.1: Consider $\operatorname{MFG}\left(K, m_{0}\right)$ under the assumptions (H1), (H3), and (H4) Let $Q \in \mathscr{P}(C(\bar{\Omega}))$ be an equilibrium of $\operatorname{MFG}\left(K, m_{0}\right), m_{t}=e_{t \#} Q$ for $t \geq 0, k$ be defined from $K$ by $k(t, x)=K\left(m_{t}, x\right)$, and $\varphi$ be the value function of $\operatorname{OCP}(k)$. Then, $\left(m_{t}, \varphi\right)$ solves the MFG system

$$
\begin{cases}\partial_{t} m_{t}-\operatorname{div}\left(m_{t} K\left(m_{t}, x\right) \widehat{\nabla \varphi}\right)=0 & \text { in } \mathbb{R}_{+}^{*} \times(\bar{\Omega} \backslash \Gamma), \\ -\partial_{t} \varphi+|\nabla \varphi| K\left(m_{t}, x\right)-1=0 & \text { in } \mathbb{R}_{+} \times(\bar{\Omega} \backslash \Gamma), \\ \varphi=0 & \text { on } \mathbb{R}_{+} \times \Gamma, \\ \nabla \varphi \cdot \mathbf{n} \geq 0 & \text { on } \mathbb{R}_{+} \times(\partial \Omega \backslash \Gamma),\end{cases}
$$

where the first equation is satisfied in the sense of distributions and the second and fourth equations are satisfied in the viscosity senses of Propositions 2.11(d) and 3.4 respectively. In addition, $\left.m_{t}\right|_{t=0}=m_{0}$ and $\left.m_{t}\right|_{\partial \Omega_{t}^{-}}=0$, where $\partial \Omega_{t}^{-}$is the part of $\partial \Omega$ at which $\widehat{\nabla \varphi} \cdot \mathbf{n}>0$.

\section{REFERENCES}

[1] M. Huang, P. E. Caines, and R. P. Malhamé, "Individual and mass behaviour in large population stochastic wireless power control problems: centralized and Nash equilibrium solutions," in 42nd IEEE
Conference on Decision and Control, 2003. Proceedings, vol. 1. IEEE, 2003, pp. 98-103.

[2] M. Huang, P. E. Caines, and R. P. Malhamé, "Large-population cost-coupled LQG problems with nonuniform agents: individual-mass behavior and decentralized $\varepsilon$-Nash equilibria," IEEE Trans. Automat. Control, vol. 52, no. 9, pp. 1560-1571, 2007.

[3] J.-M. Lasry and P.-L. Lions, "Jeux à champ moyen. I. Le cas stationnaire," C. R. Math. Acad. Sci. Paris, vol. 343, no. 9, pp. 619$625,2006$.

[4] — , "Jeux à champ moyen. II. Horizon fini et contrôle optimal," $C$. R. Math. Acad. Sci. Paris, vol. 343, no. 10, pp. 679-684, 2006.

[5] R. J. Aumann and L. S. Shapley, Values of non-atomic games. Princeton University Press, Princeton, N.J., 1974.

[6] B. Jovanovic and R. W. Rosenthal, "Anonymous sequential games," J. Math. Econom., vol. 17, no. 1, pp. 77-87, 1988.

[7] R. Carmona and F. Delarue, Probabilistic theory of mean field games with applications. I and II. Springer, Cham, 2018.

[8] E. Cristiani, B. Piccoli, and A. Tosin, Multiscale modeling of pedestrian dynamics. Springer, Cham, 2014, vol. 12.

[9] L. Gibelli and N. Bellomo, Eds., Crowd Dynamics, Volume 1. Springer International Publishing, 2018.

[10] A. Muntean and F. Toschi, Eds., Collective dynamics from bacteria to crowds. Springer, Vienna, 2014, vol. 553.

[11] F. Bagagiolo, S. Faggian, R. Maggistro, and R. Pesenti, "Optimal control of the mean field equilibrium for a pedestrian tourists' flow model," Networks and Spatial Economics, jul 2019.

[12] M. Burger, M. Di Francesco, P. A. Markowich, and M.-T. Wolfram, "On a mean field game optimal control approach modeling fast exit scenarios in human crowds," in 52nd IEEE Conference on Decision and Control. IEEE, dec 2013.

[13] S. Dweik and G. Mazanti, "Sharp semi-concavity in a non-autonomous control problem and $L^{p}$ estimates in an optimal-exit MFG," NoDEA Nonlinear Differential Equations Appl., vol. 27, no. 2, pp. Paper No. 11, 59 pp., 2020.

[14] A. Lachapelle and M.-T. Wolfram, "On a mean field game approach modeling congestion and aversion in pedestrian crowds," Transportation Research Part B: Methodological, vol. 45, no. 10, pp. 1572-1589, dec 2011.

[15] G. Mazanti and F. Santambrogio, "Minimal-time mean field games," Math. Models Methods Appl. Sci., vol. 29, no. 8, pp. 1413-1464, 2019.

[16] S. Sadeghi Arjmand and G. Mazanti, "Multi-population minimal-time mean field games," arXiv:2103.12668.

[17] P. Cannarsa and M. Castelpietra, "Lipschitz continuity and local semiconcavity for exit time problems with state constraints," J. Differential Equations, vol. 245, no. 3, pp. 616-636, 2008.

[18] P. Cannarsa and C. Sinestrari, Semiconcave functions, Hamilton-Jacobi equations, and optimal control. Birkhäuser Boston, Inc., Boston, MA, 2004, vol. 58.

[19] F. H. Clarke, Optimization and nonsmooth analysis, 2nd ed. Society for Industrial and Applied Mathematics (SIAM), Philadelphia, PA, 1990, vol. 5 .

[20] P. Cannarsa, M. Castelpietra, and P. Cardaliaguet, "Regularity properties of attainable sets under state constraints," in Geometric control and nonsmooth analysis. World Sci. Publ., Hackensack, NJ, 2008, vol. 76, pp. 120-135.

[21] P. Cannarsa and R. Capuani, "Existence and uniqueness for mean field games with state constraints," in PDE models for multi-agent phenomena. Springer, Cham, 2018, vol. 28, pp. 49-71.

[22] P. Cannarsa, R. Capuani, and P. Cardaliaguet, " $C^{1,1}$-smoothness of constrained solutions in the calculus of variations with application to mean field games," Math. Eng., vol. 1, no. 1, pp. 174-203, 2019.

[23] - ,Mean field games with state constraints: from mild to pointwise solutions of the PDE system," arXiv:1812.11374.

[24] M. Bardi and I. Capuzzo-Dolcetta, Optimal control and viscosity solutions of Hamilton-Jacobi-Bellman equations. Birkhäuser Boston, Inc., Boston, MA, 1997.

[25] P. Cardaliaguet, "Weak solutions for first order mean field games with local coupling," in Analysis and geometry in control theory and its applications. Springer, Cham, 2015, vol. 11, pp. 111-158.

[26] P. Cardaliaguet, A. R. Mészáros, and F. Santambrogio, "First order mean field games with density constraints: pressure equals price," SIAM J. Control Optim., vol. 54, no. 5, pp. 2672-2709, 2016.

[27] M. C. Delfour and J.-P. Zolésio, "Shape analysis via oriented distance functions," J. Funct. Anal., vol. 123, no. 1, pp. 129-201, 1994. 